RESEARCH ARTICLE

\title{
Field Evaluation of Thiamethoxam 25 WG Against Sucking Pests in Okra
}

\author{
Karthik P*, Vinothkumar B and Kuttalam S
}

Department of Agricultural Entomology, Tamil Nadu Agricultural University, Coimbatore-641 003

Received : 03 ${ }^{\text {rd }}$ July, 2020

Revised : $31^{\text {st }}$ July, 2020

Revised : $13^{\text {th }}$ August, 2020

Accepted : $03^{\text {rd }}$ September, 2020

\begin{abstract}
Two field experiments were conducted to test the efficacy of different doses of thiamethoxam against okra sucking pests viz., Aphids, Aphis gossypii; leaf hopper, Amrasca biguttela biguttela, and whitefly, Bemisia tabaci and their predators in okra agroecosystem. Thiamethoxam 25\% WG 25 g a.i./ha (84.71-91.73, 94.12 - 98.11\% reduction over control) was highly effective against aphid, whitefly and leaf hoppers which was on par with $50 \mathrm{~g}$ a.i./ ha $(64.28$ - 76.90, 83.70 - 87.92\% reduction over control) and 75 g a.i./ ha (73.48 - 81.26 and 85.26-92.42\% reduction over control) after first and second spray, respectively. This was further followed by standard check thiamethoxam 25\% WG @ 25 g a.i./ha (Willoxam $®)$ ). The plots treated with Thiamethoxam 25\% WG @ 25, 50 and $75 \mathrm{~g}$ a.i. /ha recorded the maximum population of Coccinella spp recorded (4.22-5.10 and 4.33-6.21 nos.) after first and second spray. Thiamethoxam 25\% WG @ 75 g a.i./ha treated plot recorded the maximum yield $\left(8100 \mathrm{~kg} / \mathrm{ha}^{-1}\right)$ followed by thiamethoxam 25\%WG @ 25 and 50 g a.i./ha (7345 and 7690.5 kg/ha-1).
\end{abstract}

Keywords: Thiamethoxam; Bioefficacy; Sucking pests; Natural enemies; Phytotoxicity.

\section{INTRODUCTION}

Okra is a vegetable crop predominantly grown in tropics and subtropics. In India, this vegetable is called as "Bhendi". Okra is good source of nutritional values and health benefits. The stem of the plant is used for the extraction of the fibre. Okra farming is very profitable and can be cultivated throughout the year. Okra is a source of higher fiber and good source of folate content. It contains vitamin A,B,K,C and excellent source of iodine. India is the largest producer of okra in Asia as well as in the world. In India, major okra growing states are Uttar Pradesh, Bihar and West Bengal. India rank first in the world with 5,784 thousand tones $(72 \%$ of the total world production) of okra. In India Andhra Pradesh is the leading okra producing state which has production of around 1184.2 thousand tons from an area of 78.90 thousand ha, which a productivity of 15 ton/ ha. Its followed by West Bengal and Bihar, 882.39, 762.90 thousand tons from an area of 75.45 and $57.71 /$ ha, respectively (Horticultural statistics at a glance,2015).

However, one of the major constrains for okra production is heavy infestations caused by several insect pests which not only exert quantitative loss but also qualitative loss to the crops. The occurrence and intensity of damage caused by them varies from different crop growth stages, regions and seasons. Again, infestations by sucking pests not only affect the crop but also hamper the crop health by transmitting pathogenic diseases.

The increasing use of conventional insecticides resulted in the development of resistance by the target insects and hazard to water, soil, environment and human health. This has led to the development of large numbers of new active compounds such as the neonicotinoids which were introduced as an alternative to conventional insecticides. Neonicotinoids have been the fastest growing class of insecticides in modern crop protection with broad spectrum effect against sucking and certain chewing insect pests. These chemical insecticides act agonistically on insect Nicotinic Acetylcholine Receptors ( $\mathrm{AAChR}$ ), blocking the nicotinergic neural pathway causing accumulation of the neurotransmitter acetylcholine Pawar et al., (2016) reported that, imidacloprid and thiamethoxam were effective in controlling the population of aphids, jassids, and whiteflies on okra. Sagar Anand Pandey (2018) found that thiamethoxam 25 WG @ 0.006\% was the most effective against aphids, leaf hopper and whitefly followed by Lambda cyhalothrin 5 EC @ 0.004\%.

The present day need emphasizes not only the use of different groups of chemicals that are eco-friendly but also give satisfactory control of 
insect pest population by their novel mode of action with minimum hazards to mammals and natural enemies. Though many formulations of thiamethoxam are available in the market and found efficacious against sucking pests, the research was carried out to investigate the newer indigenous formulation of thiamethoxam 25 WG developed by Crystal Crop Protection Pvt. Ltd. New Delhi against sucking insects in okra.

\section{MATERIAL AND METHODS}

Two field experiments were conducted one at Madhampatti and second at Narasima nayakkanpalayam to test the efficacy of different doses of thiamethoxam against okra sucking pest of okra viz., Aphids, Aphis gossypii; leaf hopper, Amrasca biguttela biguttela, and whitefly, Bemisia tabaci. The experiments were conducted using RBD with six treatments replicated four times with a plot size of $4 \times 5 \mathrm{~m}$ using the variety Okra / Ajeet-333. The treatments for the management of sucking pests comprised of thiamethoxam 25 WG @ 15, 25, 50, 75 and commercial formulation of thiamethoxam 25 WG (willoxam) @ 25 g a.i.ha-1. An untreated control was simultaneously maintained during the study. The treatments were imposed twice at fifteen days interval commencing from $30^{\text {th }}$ day after planting with pneumatic knapsack sprayer using 500 liters of spray fluid per hectare. The average temperature in the open field during the study period was 27 $31{ }^{\circ} \mathrm{C}$ with relative humidity between 70 and $80 \%$ and there was no rainfall during the periods of observation.

The pre and post treatment observations on third, seventh and fourteenth days after treatment (DAT) were recorded on the incidence of aphids, leaf hopper and whitefly. The observations were made on three leaves/ plant, one each from top, middle and bottom regions from five plants per plot selected at random leaving border rows on before imposing treatments, 3, 7 and 14 days after spraying. The mean number of pests and per cent reduction over control were calculated for each spray. Yield also recorded from each picking for the various treatments and were pooled and expressed in tonnes ha-1.

To evaluate the phytotoxicity (if any) by thiamethoxam $25 \%$ WG on okra, experiments were conducted one at Madhampatti and second at Narasimanayakkanpalayam (cultivar-Ajeet) on the same season. Three doses were tested at 25 , 50 and $100 \mathrm{~g}$ a.i.ha ${ }^{-1}$ Observations were recorded in radmoly selected five plants per plot. To know the crop tolerance, the plants were observed on 1, 3, 7 , 10 and 14 days after spraying as per the protocol of Central Insecticide Board Registration Committee (C.I.B. and R.C) for the phytotoxic symptoms like leaf tip, wilting, necrosis, vein clearing, epinasty and hyponasty. The per cent leaf injury was calculated using the following formula,

$$
\begin{aligned}
& \text { Percent leaf } \\
& \text { injury }
\end{aligned}=\frac{\text { Total grade points }}{\begin{array}{c}
\text { Maximum grade } X \text { Number of } \\
\text { leaves observed }
\end{array}} \times 100
$$

The phytotoxicity symptoms, if any, were graded based on the per cent injured leaves as per the C.I.B and R.C). grade scale viz., no phytotoxicity - grade $0 ; 1-10 \%$-grade $1 ; 11-20 \%$ grade $2 ; 21-30 \%$ grade $3 ; 31-40 \%$ grade $4 ; 41-50 \%$ grade $5 ; 51-60 \%$ grade $6 ; 61-70 \%$ grade $7 ; 71-80 \%$ grade $8 ; 81-90 \%$ grade 9; $91-100 \%$ grade 10.

Ten randomly tagged plants per plot were thoroughly observed for population of natural enemies. The population of natural enemies was recorded based on number of adult of coccinellids. The observations were taken before and 3, 7 and 14 days after each spraying.

\section{RESULTS AND DISCUSSION}

The aphid population prior to first spraying was 20.17 to 23.50 per three leaves (Table.1). Thiamethoxam 25\% WG at the rate of 75 ga.i.ha ${ }^{-1}$ reduced the mean aphid population significantly (4.35 nos. per three leaves/plant) with 91.73 per cent reduction over control and it was on par with thiamethoxam 25\% WG at 50 and 25 ga.i.ha ${ }^{-1}$ (4.35 and 4.90 nos. per three leaves/plant) (Fig.1)

The standard check, Willoxam ${ }^{\circledR}$ at 25 g a.i. ha ${ }^{-1}$ recorded the mean aphid population of 5.24 nos. per three leaves/plant when compared to untreated check (5.24 nos. per three leaves/plant). Similar trend was observed during the second spray. Thiamethoxam $25 \%$ WG at $75 \mathrm{~g}$ a.i. ha ${ }^{-1}$ recorded least population (0.63 nos. per three leaves/plant) of aphids with 98.11 per cent reduction over control. The standard check, Willoxam ${ }^{\circledR}$ at the rate of $25 \mathrm{~g}$ a.i. ha ${ }^{-1}$ recorded 93.25 per cent reduction over control. Whereas, untreated check recorded the population of 33.35 nos. per three leaves/plant. The result of the current study is in accordance with the report of Pawar et al., (2016), who demonstrated the effectiveness of thiamethoxam 25\% WG against the sucking pests of okra.

The mean data revealed that the highest dose of thiamethoxam @ 75 g a.i.ha ${ }^{-1}$ recorded minimum mean whitefly population of 2.91 and 0.87 nos. per three leaves/ plant, after the first, and second spray respectively as against untreated check (12.60 and 10.68 per three leaves/plant) and their corresponding per cent reduction over control was 76.90 and 91.85 (Fig.1). The standard check, Willoxam ${ }^{\circledR}$ at the rate of $25 \mathrm{~g}$ a.i.ha-1 ${ }^{-1}$ recorded the mean population of 4.83 and 2.14 nos. per three leaves / plant, respectively after the first and second 
Table 1. Effect of thiamethoxam 25 WG on aphids in okra (Pooled mean of two seasons)

\begin{tabular}{|c|c|c|c|c|c|c|c|c|c|c|c|c|}
\hline \multirow{3}{*}{ S.No } & \multirow{3}{*}{ Treatments } & \multirow{3}{*}{ PTC } & \multicolumn{4}{|c|}{ First spray } & \multirow{3}{*}{$\begin{array}{l}\text { Percent } \\
\text { reduction } \\
\text { over } \\
\text { check }\end{array}$} & \multirow{2}{*}{\multicolumn{4}{|c|}{$\begin{array}{c}\text { Second spray } \\
\text { Number/ } 3 \text { leaves/ plant* }\end{array}$}} & \multirow{3}{*}{$\begin{array}{l}\text { Percent } \\
\text { reduction over } \\
\text { check }\end{array}$} \\
\hline & & & \multicolumn{4}{|c|}{ Number/ 3 leaves/ plant* } & & & & & & \\
\hline & & & 3 DAT & 7 DAT & 14 DAT & Mean & & 3 DAT & 7 DAT & 14 DAT & Mean & \\
\hline 1. & $\begin{array}{l}\text { Thiamethoxam } 25 \% \text { WG } \\
\text { @15 g a.i ha }{ }^{-1}\end{array}$ & 20.63 & $\begin{array}{r}9.57^{\mathrm{c}} \\
(3.16)\end{array}$ & $\begin{array}{l}7.37^{\mathrm{bc}} \\
(2.80)\end{array}$ & $\begin{array}{l}5.10^{\mathrm{bc}} \\
(2.37)\end{array}$ & 7.34 & 72.09 & $\begin{array}{r}4.07^{\mathrm{b}} \\
(2.13)\end{array}$ & $\begin{array}{r}3.87^{\mathrm{b}} \\
(2.09)\end{array}$ & $\begin{array}{r}3.93^{\mathrm{b}} \\
(2.10)\end{array}$ & 3.95 & 88.15 \\
\hline 2. & $\begin{array}{l}\text { Thiamethoxam } 25 \text { \% } \\
\text { WG@25 g a.i ha }{ }^{-1}\end{array}$ & 21.43 & $\begin{array}{l}7.20^{\mathrm{bc}} \\
(2.77)\end{array}$ & $\begin{array}{r}5.07^{\mathrm{b}} \\
(2.36)\end{array}$ & $\begin{array}{l}2.43^{\mathrm{ab}} \\
(1.69)\end{array}$ & 4.90 & 84.71 & $\begin{array}{l}2.03^{\mathrm{ab}} \\
(1.58)\end{array}$ & $\begin{array}{r}1.90^{\mathrm{b}} \\
(1.54)\end{array}$ & $\begin{array}{l}1.97^{\mathrm{ab}} \\
(1.57)\end{array}$ & 1.96 & 94.12 \\
\hline 3. & $\begin{array}{l}\text { Thiamethoxam } 25 \text { \% } \\
\text { WG@50g a.i ha-1 }\end{array}$ & 20.17 & $\begin{array}{l}6.23^{\mathrm{ab}} \\
(2.59)\end{array}$ & $\begin{array}{l}4.53^{\mathrm{ab}} \\
(2.24)\end{array}$ & $\begin{array}{r}2.30^{\mathrm{a}} \\
(1.41)\end{array}$ & 4.35 & 86.42 & $\begin{array}{l}1.97^{\mathrm{ab}} \\
(1.56)\end{array}$ & $\begin{array}{l}1.73^{\mathrm{ab}} \\
(1.47)\end{array}$ & $\begin{array}{l}1.80^{\mathrm{ab}} \\
(1.50)\end{array}$ & 1.83 & 94.51 \\
\hline 4. & $\begin{array}{l}\text { Thiamethoxam } 25 \text { \% } \\
\text { WG@75g a.i ha }{ }^{-1}\end{array}$ & 23.50 & $\begin{array}{r}4.37 \mathrm{a} \\
(2.16)\end{array}$ & $\begin{array}{r}2.37^{\mathrm{a}} \\
(1.67)\end{array}$ & $\begin{array}{r}1.23^{\mathrm{a}} \\
(1.30)\end{array}$ & 2.65 & 91.73 & $\begin{array}{r}0.70^{\mathrm{a}} \\
(1.09)\end{array}$ & $\begin{array}{r}0.57^{\mathrm{a}} \\
(1.03)\end{array}$ & $\begin{array}{r}0.63^{\mathrm{a}} \\
(1.06)\end{array}$ & 0.63 & 98.11 \\
\hline 5. & $\begin{array}{l}\text { Thiamethoxam } 25 \% \text { WG } \\
@ 25 \text { g a.i ha }^{-1} \text { (Willoxam) }\end{array}$ & 22.87 & $\begin{array}{l}7.70^{\mathrm{bc}} \\
(2.85)\end{array}$ & $\begin{array}{r}5.53^{\mathrm{b}} \\
(2.37)\end{array}$ & $\begin{array}{l}2.50^{\mathrm{ab}} \\
(1.65)\end{array}$ & 5.24 & 83.65 & $\begin{array}{l}2.43^{\mathrm{ab}} \\
(1.71)\end{array}$ & $\begin{array}{l}2.10^{\mathrm{ab}} \\
(1.61)\end{array}$ & $\begin{array}{l}2.23^{\mathrm{ab}} \\
(1.65)\end{array}$ & 2.25 & 93.25 \\
\hline 6. & Untreated check & 20.73 & $\begin{array}{c}34.37^{\mathrm{d}} \\
(5.90)\end{array}$ & $\begin{array}{c}31.90^{\mathrm{d}} \\
(5.69)\end{array}$ & $\begin{array}{c}29.90^{\circ} \\
(5.51)\end{array}$ & 32.05 & - & $\begin{array}{c}32.50^{\circ} \\
(5.74)\end{array}$ & $\begin{array}{c}34.27^{\circ} \\
(5.90)\end{array}$ & $\begin{array}{c}33.30^{\circ} \\
(5.81)\end{array}$ & 33.35 & \\
\hline
\end{tabular}

PTC - Pre treatment count; DAT - Days after treatment ; *Mean of four replications Figures in parentheses are $\sqrt{x+0.5}$ transformed values In a column, means followed by a common letter(s) are not significantly different by DMRT $(p=0.05)$

spray (Tables 2). Awaneesh Kumar et al.(2017) reported that Thiamethoxam 25WG @ 100g/ha-1 was found most effective insecticide in reducing the population of whitefly followed byimidacloprid 17.8 SL @ 100 ml/ha. Prem Kumar and Ashwani
Kumar (2017) found that Imidiacloprid 17.8SL was the most effective treatment indicating reduction in population of whitefly (1.33/3leaves) and jassid (1.26/3leaves), followed by Thiamethoxam 25WG.

Table 2. Effect of thiamethoxam 25 WG on whiteflies in okra (Pooled mean of two seasons)

\begin{tabular}{|c|c|c|c|c|c|c|c|c|c|c|c|c|}
\hline \multirow{3}{*}{ S.No } & \multirow{3}{*}{ Treatments } & \multirow{3}{*}{ PTC } & \multicolumn{4}{|c|}{ First spray } & \multirow{3}{*}{$\begin{array}{l}\text { Percent } \\
\text { reduction } \\
\text { over } \\
\text { check }\end{array}$} & \multirow{2}{*}{\multicolumn{4}{|c|}{$\begin{array}{c}\text { Second spray } \\
\text { Number/ } 3 \text { leaves/ plant* }\end{array}$}} & \multirow{3}{*}{$\begin{array}{l}\text { Percent } \\
\text { reduction } \\
\text { over } \\
\text { check }\end{array}$} \\
\hline & & & \multicolumn{4}{|c|}{ Number/ 3 leaves/ plant* } & & & & & & \\
\hline & & & 3 DAT & 7 DAT & 14 DAT & Mean & & 3 DAT & 7 DAT & 14 DAT & Mean & \\
\hline 1. & $\begin{array}{l}\text { Thiamethoxam } 25 \text { \% } \\
\text { WG @15 g a.i ha }{ }^{-1}\end{array}$ & 12.47 & $\begin{array}{r}4.97 \\
(2.23)^{\mathrm{b}}\end{array}$ & $\begin{array}{r}5.20 \\
(2.28)^{\mathrm{b}}\end{array}$ & $\begin{array}{r}6.50 \\
(2.55)^{\mathrm{c}}\end{array}$ & 5.55 & 55.95 & $\begin{array}{r}2.73 \\
(1.65)^{\mathrm{b}}\end{array}$ & $\begin{array}{r}2.73 \\
(1.65)^{\mathrm{b}}\end{array}$ & $\begin{array}{r}2.20 \\
(1.48)^{\mathrm{b}}\end{array}$ & 2.55 & 76.12 \\
\hline 2. & $\begin{array}{l}\text { Thiamethoxam } 25 \% \\
\text { WG @25 g a.i ha-1 }\end{array}$ & 12.00 & $\begin{array}{r}4.07 \\
(2.02)^{\mathrm{ab}}\end{array}$ & $\begin{array}{r}4.13 \\
(2.03)^{\mathrm{ab}}\end{array}$ & $\begin{array}{r}5.30 \\
(2.30)^{\mathrm{bc}}\end{array}$ & 4.50 & 64.28 & $\begin{array}{r}2.00 \\
(1.41)^{\mathrm{ab}}\end{array}$ & $\begin{array}{r}1.80 \\
(1.34)^{\mathrm{ab}}\end{array}$ & $\begin{array}{r}1.43 \\
(1.20)^{\mathrm{ab}}\end{array}$ & 1.74 & 83.70 \\
\hline 3. & $\begin{array}{l}\text { Thiamethoxam } 25 \% \\
\text { WG @50g a.i ha-1 }\end{array}$ & 11.53 & $\begin{array}{r}3.47 \\
(1.86)^{\mathrm{ab}}\end{array}$ & $\begin{array}{r}3.53 \\
(1.88)^{\mathrm{ab}}\end{array}$ & $\begin{array}{r}4.90 \\
(2.21)^{\mathrm{ab}}\end{array}$ & 3.96 & 68.57 & $\begin{array}{r}1.73 \\
(1.32)^{\mathrm{ab}}\end{array}$ & $\begin{array}{r}1.33 \\
(1.15)^{\mathrm{ab}}\end{array}$ & $\begin{array}{r}0.83 \\
(0.91)^{\text {ab }}\end{array}$ & 1.29 & 87.92 \\
\hline 4. & $\begin{array}{l}\text { Thiamethoxam } 25 \% \\
\text { WG @75g a.i ha-1 }\end{array}$ & 12.10 & $\begin{array}{r}2.07 \\
(1.44)^{\mathrm{a}}\end{array}$ & $\begin{array}{r}3.27 \\
(1.81)^{\mathrm{a}}\end{array}$ & $\begin{array}{r}3.40 \\
(2.10)^{\mathrm{a}}\end{array}$ & 2.91 & 76.90 & $\begin{array}{r}1.33 \\
(1.15)^{\mathrm{a}}\end{array}$ & $\begin{array}{r}1.00 \\
(1.00)^{\mathrm{a}}\end{array}$ & $\begin{array}{r}0.30 \\
(0.55)^{\mathrm{a}}\end{array}$ & 0.87 & 91.85 \\
\hline 5. & $\begin{array}{l}\text { Thiamethoxam } 25 \\
\text { \% WG @ } 25 \text { g a.i ha-1 } \\
\text { (Willoxam) }\end{array}$ & 12.90 & $\begin{array}{r}4.40 \\
(2.10)^{\mathrm{ab}}\end{array}$ & $\begin{array}{r}4.40 \\
(2.10)^{\text {ab }}\end{array}$ & $\begin{array}{r}5.70 \\
(2.39)^{\mathrm{bc}}\end{array}$ & 4.83 & 61.66 & $\begin{array}{r}2.27 \\
(1.51)^{\mathrm{ab}}\end{array}$ & $\begin{array}{r}2.47 \\
(1.57)^{\text {ab }}\end{array}$ & $\begin{array}{r}1.70 \\
(1.30)^{\mathrm{ab}}\end{array}$ & 2.14 & 79.96 \\
\hline 6. & Untreated check & 12.93 & $\begin{array}{r}14.33 \\
(3.65)^{\mathrm{c}}\end{array}$ & $\begin{array}{l}12.47 \\
(3.53)^{\mathrm{c}}\end{array}$ & $\begin{array}{r}11.00 \\
(3.32)^{d}\end{array}$ & 12.60 & - & $\begin{array}{r}10.59 \\
(3.25)^{\mathrm{c}}\end{array}$ & $\begin{array}{r}11.23 \\
(3.35)^{\mathrm{c}}\end{array}$ & $\begin{array}{r}10.23 \\
(3.20)^{c}\end{array}$ & 10.68 & - \\
\hline
\end{tabular}

PTC - Pre treatment count; DAT - Days after treatment ; *Mean of four replications Figures in parentheses are $\sqrt{x+0.5}$ transformed values

In a column, means followed by a common letter(s) are not significantly different by DMRT ( $\mathrm{p}=0.05)$

The mean population of leafhoppers prior to first spraying was 7.00 to 8.20 per three leaves plant ${ }^{-1}$ (Table 3). Thiamethoxam 25\% WG at the rate of 75 ga.i.ha-1 reduced the mean leaf hopper population significantly (2.17 nos. per three leaves/plant) with 81.26 per cent reduction over control followed by thiamethoxam 25\% WG at 50 (2.90 nos. per three leaves/plant). The standard check, Willoxam ${ }^{\circledR}$ at the rate of $25 \mathrm{~g}$ a.i. ha ${ }^{-1}$ recorded the population of 3.34 nos. per three leaves/plant when compared to untreated check (11.58 nos. per three leaves/ plant). Aarwe Rajesh et al.(2015) revealed that mean population of jassid was least in the plots treated with tiamethoxam 25\%WG @ 50 g a.i./ha followed by tiamethoxam 25 \% WG @ 25 a.i./ha. Ghosh et al.(2016) reported that thiamethoxam 25\% WG @ 50 g a.i. ha ${ }^{-1}$ was highly effective against sucking pests of okra in Terai region of West Bengal. Gaikwad et al., (2014) reported that, thiamethoxam 25WG @ 75 g

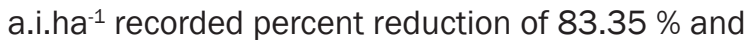
$96.67 \%$ of Jassids, $92.95 \%$ and $99.47 \%$ of aphids and $83.80 \%$ and $96.67 \%$ of White flies, respectively in first and second spray. Manju et al. (2018) reported that the lowest okra yellow vein mosaic virus incidence was reported in thiamethoxam 70 WS (16.56\%) followed by imidacloprid 70 WS (16.90 $\%)$ and spinosad 45 SC (19.43\%).

Similar trend was observed during the second spray. Thiamethoxam $25 \%$ WG at the rate of $75 \mathrm{~g}$ a.i. ha $^{-1}$ recorded higher reduction in aphid population (0.93 nos. per three leaves/plant) with 92.42 per cent reduction over control (Fig.1). 
Table 3. Effect of thiamethoxam 25 WG on leaf hopper (Pooled mean of two seasons)

\begin{tabular}{|c|c|c|c|c|c|c|c|c|c|c|c|c|c|}
\hline \multirow{3}{*}{ S.No } & \multirow{3}{*}{ Treatments } & \multirow{3}{*}{ PTC } & \multicolumn{4}{|c|}{ First spray } & \multirow{3}{*}{$\begin{array}{l}\text { Percent } \\
\text { reduction } \\
\text { over } \\
\text { check }\end{array}$} & \multirow{2}{*}{\multicolumn{4}{|c|}{$\begin{array}{c}\text { Second spray } \\
\text { Number/ } 3 \text { leaves/ plant* }\end{array}$}} & \multirow{3}{*}{$\begin{array}{l}\text { Percent } \\
\text { reduction } \\
\text { over check }\end{array}$} & \multirow{3}{*}{$\begin{array}{l}\text { Yield* } \\
\text { (Kg/ha) }\end{array}$} \\
\hline & & & \multicolumn{4}{|c|}{ Number/ 3 leaves/ plant* } & & & & & & & \\
\hline & & & 3 DAT & 7 DAT & $\begin{array}{c}14 \\
\text { DAT }\end{array}$ & Mean & & 3 DAT & 7 DAT & $\begin{array}{c}14 \\
\text { DAT }\end{array}$ & Mean & & \\
\hline 1. & $\begin{array}{l}\text { Thiamethoxam } 25 \text { \% WG @15 } \\
\text { g a.i ha }{ }^{-1}\end{array}$ & 12.47 & $\begin{array}{r}4.83 \\
(2.20)^{\mathrm{b}}\end{array}$ & $\begin{array}{r}2.50 \\
(1.58)^{\mathrm{b}}\end{array}$ & $\begin{array}{r}4.40 \\
(2.10)^{\mathrm{b}}\end{array}$ & 3.91 & 66.23 & $\begin{array}{r}3.50 \\
(1.87)^{\circ}\end{array}$ & $\begin{array}{r}2.00 \\
(1.41)^{\mathrm{b}}\end{array}$ & $\begin{array}{r}1.27 \\
(1.13)^{\mathrm{b}}\end{array}$ & 2.25 & 81.67 & $5726.50^{c}$ \\
\hline 2. & $\begin{array}{l}\text { Thiamethoxam 25 \% WG @25 } \\
\text { g a.i ha }{ }^{-1}\end{array}$ & 12.00 & $\begin{array}{r}3.40 \\
(1.84)^{\mathrm{ab}}\end{array}$ & $\begin{array}{r}2.23 \\
(1.49)^{\mathrm{ab}}\end{array}$ & $\begin{array}{r}3.60 \\
(1.90)^{\mathrm{b}}\end{array}$ & 3.07 & 73.48 & $\begin{array}{r}2.93 \\
(1.71)^{\mathrm{bc}}\end{array}$ & $\begin{array}{r}1.50 \\
(1.22)^{\mathrm{ab}}\end{array}$ & $\begin{array}{r}1.00 \\
(1.00)^{\mathrm{ab}}\end{array}$ & 1.81 & 85.26 & $7345.00^{b}$ \\
\hline 3. & $\begin{array}{l}\text { Thiamethoxam } 25 \text { \% WG } \\
\text { @50g a.i ha }{ }^{1}\end{array}$ & 11.53 & $\begin{array}{r}3.17 \\
(1.78)^{\mathrm{ab}}\end{array}$ & $\begin{array}{r}2.00 \\
(1.41)^{\mathrm{ab}}\end{array}$ & $\begin{array}{r}3.53 \\
(1.88) \\
a b\end{array}$ & 2.90 & 74.95 & $\begin{array}{r}2.73 \\
(1.65)^{\mathrm{b}}\end{array}$ & $\begin{array}{r}1.50 \\
(1.22)^{\mathrm{ab}}\end{array}$ & $\begin{array}{r}0.80 \\
(0.89)^{\mathrm{ab}}\end{array}$ & 1.67 & 86.40 & $7690.50^{\mathrm{b}}$ \\
\hline 4. & $\begin{array}{l}\text { Thiamethoxam } 25 \text { \% WG } \\
\text { @75g a.i ha }{ }^{-1}\end{array}$ & 12.10 & $\begin{array}{r}2.63 \\
(1.62)^{\mathrm{a}}\end{array}$ & $\begin{array}{r}1.50 \\
(1.22)^{\mathrm{a}}\end{array}$ & $\begin{array}{r}2.40 \\
(1.55)^{\mathrm{a}}\end{array}$ & 2.17 & 81.26 & $\begin{array}{r}1.40 \\
(1.18)^{\mathrm{a}}\end{array}$ & $\begin{array}{r}1.00 \\
(1.00)^{a}\end{array}$ & $\begin{array}{r}0.40 \\
(0.63)^{\mathrm{a}}\end{array}$ & 0.93 & 92.42 & $8100.50^{a}$ \\
\hline 5. & $\begin{array}{l}\text { Thiamethoxam } 25 \text { \% WG @25 } \\
\text { g a.i ha }{ }^{-1} \text { (Willoxam) }\end{array}$ & 12.90 & $\begin{array}{r}3.70 \\
(1.92)^{\mathrm{ab}}\end{array}$ & $\begin{array}{r}2.00 \\
(1.41)^{\mathrm{ab}}\end{array}$ & $\begin{array}{r}4.33 \\
(2.08)^{b}\end{array}$ & 3.34 & 71.15 & $\begin{array}{r}3.13 \\
(1.77)^{\mathrm{bc}}\end{array}$ & $\begin{array}{r}1.70 \\
(1.30)^{\mathrm{ab}}\end{array}$ & $\begin{array}{r}1.13 \\
(1.06)^{\mathrm{ab}}\end{array}$ & 1.98 & 83.87 & $6915.00^{\text {bc }}$ \\
\hline 6. & Untreated check & 12.93 & $\begin{array}{r}11.07 \\
(3.33)^{\mathrm{c}}\end{array}$ & $\begin{array}{r}11.50 \\
(3.39)^{c}\end{array}$ & $\begin{array}{l}12.17 \\
(3.49)^{\circ}\end{array}$ & 11.58 & - & $\begin{array}{r}11.13 \\
(2.85)^{d}\end{array}$ & $\begin{array}{r}12.50 \\
(2.92)^{\mathrm{c}}\end{array}$ & $\begin{array}{r}13.23 \\
(3.04)^{\mathrm{c}}\end{array}$ & 12.28 & - & $4936.00^{d}$ \\
\hline
\end{tabular}

PTC - Pre treatment count; DAT - Days after treatment ; * Mean of four replications Figures in parentheses are $\sqrt{x+0.5}$ transformed values In a column, means followed by a common letter(s) are not significantly different by DMRT $(p=0.05)$

The standard check, Willoxam at the rate of $25 \mathrm{~g}$ a.i. ha ${ }^{-1}$ recorded the mean aphid population of 1.98 nos. per three leaves/plant with 83.87 per cent reduction over control. The untreated check recorded the population of 12.28 nos. per three leaves/plant. Bade et al.(2017) found that acetamiprid 20 SP @ 15 g a.i./ha and thiamethoxam 25 WG @ 25 g a.i./ha were found most effective treatment against aphids after four sprays.

Table 4. Effect of thiamethoxam 25 \% WG on coccinellids in okra eco system (Pooled mean of two seasons)

\begin{tabular}{|c|c|c|c|c|c|c|c|c|c|c|c|}
\hline \multirow{3}{*}{ S.No } & \multirow{3}{*}{ Treatments } & \multirow{3}{*}{ PTC } & \multicolumn{4}{|c|}{ First spray } & \multirow{3}{*}{$\begin{array}{c}\text { Percent } \\
\text { reduction } \\
\text { over check }\end{array}$} & \multicolumn{4}{|c|}{ Second spray } \\
\hline & & & \multicolumn{4}{|c|}{ Number/ 3 leaves/ plant* } & & \multicolumn{4}{|c|}{ Number/ 3 leaves/ plant* } \\
\hline & & & 3 DAT & 7 DAT & 14 DAT & Mean & & 3 DAT & 7 DAT & 14 DAT & Mean \\
\hline 1. & $\begin{array}{l}\text { Thiamethoxam } 25 \text { \% } \\
\text { WG @15 g a.i ha-1 }\end{array}$ & 7.00 & $\begin{array}{r}5.67 \\
(2.48)^{\mathrm{b}}\end{array}$ & $\begin{array}{r}6.17 \\
(2.58)^{\mathrm{b}}\end{array}$ & $\begin{array}{r}6.93 \\
(2.72)^{\mathrm{b}}\end{array}$ & 6.25 & $\begin{array}{r}4.60 \\
(2.25)^{\mathrm{b}}\end{array}$ & $\begin{array}{r}7.13 \\
(2.76)^{\mathrm{b}}\end{array}$ & $\begin{array}{r}8.67 \\
(3.02)^{b}\end{array}$ & 6.80 & 2.55 \\
\hline 2. & $\begin{array}{l}\text { Thiamethoxam } 25 \% \\
\text { WG @25 g a.i ha-1 }\end{array}$ & 6.00 & $\begin{array}{r}4.66 \\
(2.26)^{\mathrm{bc}}\end{array}$ & $\begin{array}{r}5.00 \\
(2.33)^{\mathrm{bc}}\end{array}$ & $\begin{array}{r}5.66 \\
(2.46)^{\mathrm{bc}}\end{array}$ & 5.10 & $\begin{array}{r}3.65 \\
(2.03)^{\mathrm{bc}}\end{array}$ & $\begin{array}{r}6.66 \\
(2.67)^{\mathrm{bc}}\end{array}$ & $\begin{array}{r}8.33 \\
(2.97)^{\mathrm{b}}\end{array}$ & 6.21 & 1.74 \\
\hline 3. & $\begin{array}{l}\text { Thiamethoxam } 25 \% \\
\text { WG @50g a.i ha-1 }\end{array}$ & 6.00 & $\begin{array}{r}4.33 \\
(2.19)^{\mathrm{bc}}\end{array}$ & $\begin{array}{r}4.66 \\
(2.25)^{\mathrm{bc}}\end{array}$ & $\begin{array}{r}4.66 \\
(2.25)^{\mathrm{c}}\end{array}$ & 4.55 & $\begin{array}{r}3.35 \\
(2.00)^{\mathrm{bc}}\end{array}$ & $\begin{array}{r}5.00 \\
(2.33)^{\mathrm{bc}}\end{array}$ & $\begin{array}{r}6.66 \\
(2.67)^{\mathrm{bc}}\end{array}$ & 5.00 & 1.29 \\
\hline 4. & $\begin{array}{l}\text { Thiamethoxam } 25 \% \\
\text { WG @75g a.i ha-1 }\end{array}$ & 5.50 & $\begin{array}{r}4.00 \\
(2.11)^{\mathrm{bc}}\end{array}$ & $\begin{array}{r}4.00 \\
(2.11)^{c}\end{array}$ & $\begin{array}{r}4.66 \\
(2.27)^{\mathrm{c}}\end{array}$ & 4.22 & $\begin{array}{r}3.00 \\
(1.87)^{\mathrm{bc}}\end{array}$ & $\begin{array}{r}4.00 \\
(2.11)^{\mathrm{c}}\end{array}$ & $\begin{array}{r}6.00 \\
(2.54)^{\mathrm{c}}\end{array}$ & 4.33 & 0.87 \\
\hline 5. & $\begin{array}{l}\text { Thiamethoxam } 25 \\
\% \text { WG @25 g a.i ha }{ }^{-1} \\
\text { (Willoxam) }\end{array}$ & 6.67 & $\begin{array}{r}4.00 \\
(2.11)^{\mathrm{bc}}\end{array}$ & $\begin{array}{r}5.33 \\
(2.41)^{\mathrm{bc}}\end{array}$ & $\begin{array}{r}5.00 \\
(2.33)^{\mathrm{bc}}\end{array}$ & 4.77 & $\begin{array}{r}4.00 \\
(2.11)^{\mathrm{bc}}\end{array}$ & $\begin{array}{r}6.00 \\
(2.53)^{\mathrm{bc}}\end{array}$ & $\begin{array}{r}7.33 \\
(2.79)^{b}\end{array}$ & 5.77 & 2.14 \\
\hline 6. & Untreated check & 6.00 & $\begin{array}{r}7.33 \\
(2.97)^{\mathrm{a}}\end{array}$ & $\begin{array}{r}8.33 \\
(2.97)^{\mathrm{a}}\end{array}$ & $\begin{array}{r}9.33 \\
(3.13)^{\mathrm{a}}\end{array}$ & 8.33 & $\begin{array}{r}11.33 \\
(2.97)^{\mathrm{a}}\end{array}$ & $\begin{array}{r}12.66 \\
(3.62)^{\mathrm{a}}\end{array}$ & $\begin{array}{r}13.00 \\
(3.67)^{\mathrm{a}}\end{array}$ & 12.33 & 10.68 \\
\hline
\end{tabular}

PTC- Pre treatment count; DAT- Days after treatments, *Mean of four replications ; Values in parentheses are $\sqrt{x+0.5}$ transformed values In a column means followed by a common letter(s) are not significantly different by DMRT $(\mathrm{P}=0.05)$

Patil et al. (2014) reported that, foliar spray of thiamethoxam 25 WG @ 0.006\% was found the most effective against aphids, leaf hoppers and whitefly population. Thiamethoxam 25 WG @ 0.006\% was effective against leafhoppers population followed by Thiamethoxam 25 WG @ 0.008\%. The highest fruit yield ( $126.14 \mathrm{q} / \mathrm{ha})$ and cost benefit ratio (1:12.62) were obtained from thiamethoxamtreated plots. Nitenpyram and thiamethoxam are highly effective for controlling the piercing sucking pests (mainly aphids, mirids, thrips and whiteflies) (Wettstein et al., 2016; Zhang et al., 2016b). Karthik et al. (2015) revealed that imidacloprid 70 WG @ 25 g a.i./ha was highly effective against aphids, leaf hopper and whitefly on okra. Sagar Anand Pandey (2018) found that thiamethoxam 25 WG @ 0.006\% was the most effective against aphids, leaf hopper and whitefly followed by Lambda cyhalothrin 5 EC @ 0.004\%. Application of imidacloprid 17.8 SL @ 0.5 $\mathrm{ml} / \mathrm{I}$ was found superior over the other treatments with higher per cent reduction (85.21 per cent) of jassids, followed by acetamiprid 20 SP @ 0.5 g/l, thiamethoxam 25 WG @ 0.3 g/l on okra (Hemadri et al. (2018). Bhatt et al. (2018) reported that thiamethoxam 25\% WG@ 25 g a.i./ha, recorded 75.99 and 76.27 per cent reduction after first and second spray, respectively.

The data recorded on the yield of bhendi, on the

$107|7-9| 310$ 

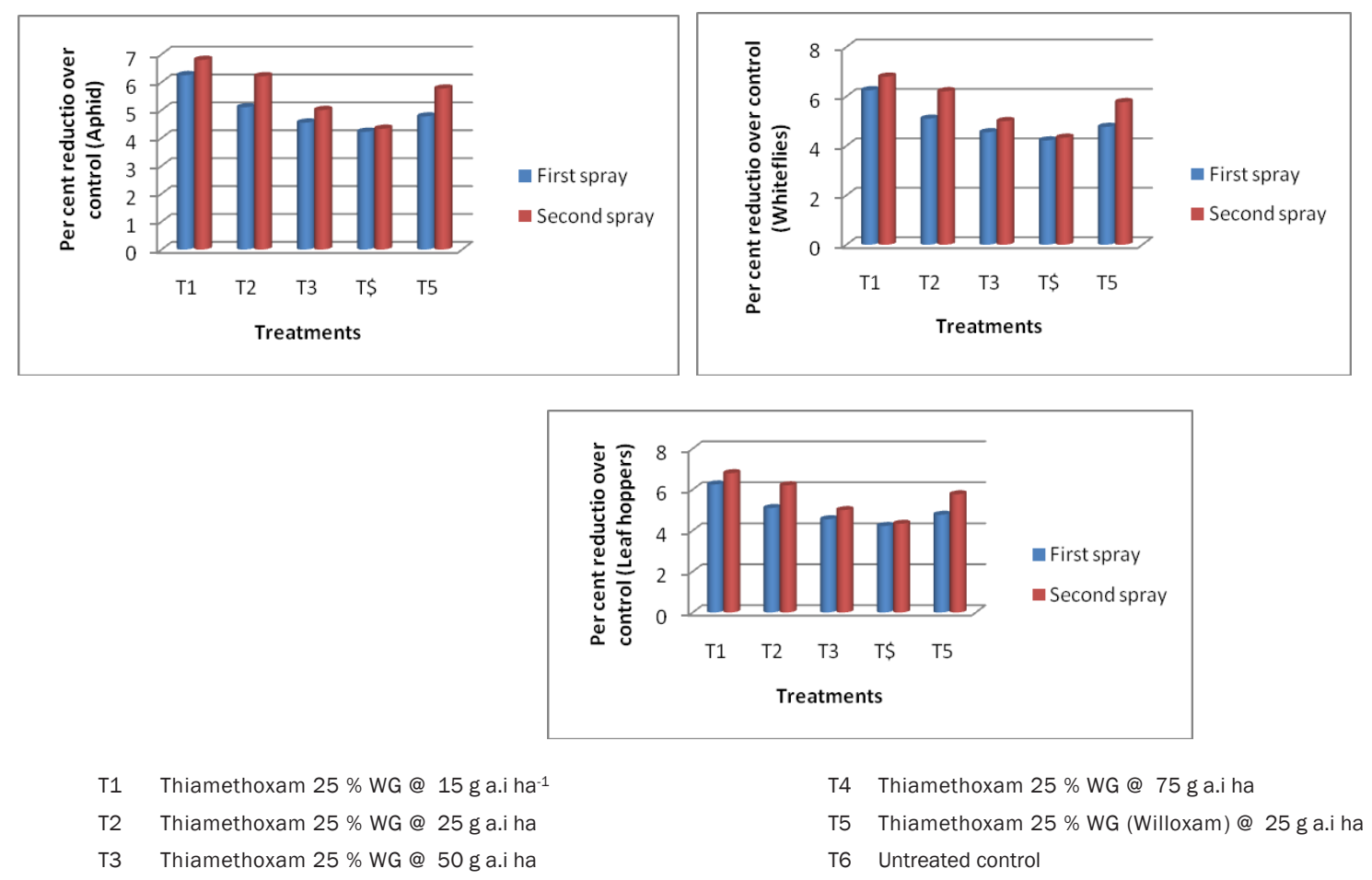

Figure 1. Effect of thiamethoxam 25 WG on aphid, whiteflies and hoppers leaf hopper

first trial indicated that thiamethoxam 25\% WG at 75 g a.i. ha-1 $\left.^{-1} 8100.50 \mathrm{~kg} \mathrm{ha}^{-1}\right)$ exhibited significantly higher yield over rest of the treatments (Table 7). thiamethoxam 25\% WG at 50 g a.i. ha ${ }^{-1}(7690.50$ $\mathrm{kg} \mathrm{ha}^{-1}$ ) was on par with thiamethoxam $25 \%$ WG at 25 g a.i. ha-1 (7345.00 kg ha-1) (Fig.2). The lower dose of thiamethoxam $25 \%$ WG at $15 \mathrm{~g}$ a.i. ha $^{-1}$ recorded yield

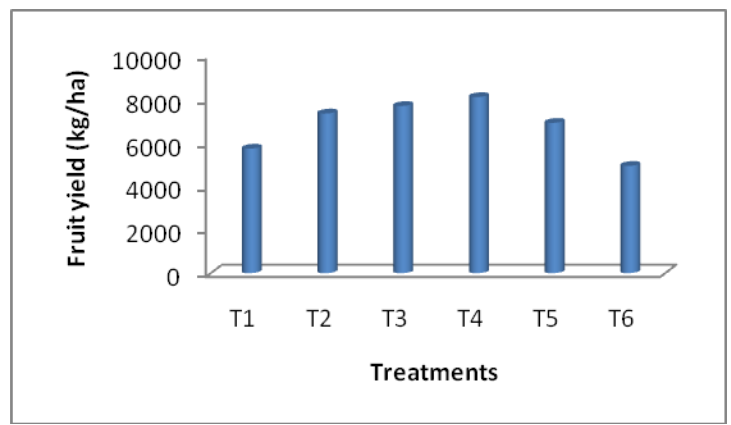

Figure 2. Impact of Insecticides on yield of okra (Pooled mean of two seasons)

of $5726.50 \mathrm{~kg} \mathrm{ha}^{-1}$. Similar results were observed by Anitha and Nandihalli (2009) who evaluated the efficacy of Thiamethoxam 25 WG $(0.2 \mathrm{~g} /$ lit.) when applied as foliar sprays and registered highest fruit yield. Similarly, Venkataravanappa et al. (2012) reported that thiamethoxam 25 WG gave highest fruit yield of okra. Babita Bhatt et al.(2018) reported that chlorantraniliprole 18.5\% SC @ 25 g a.i./ha treated plot recorded the maximum yield (10.81 T/ ha) followed by thiamethoxam 25\%WG @ 25 ga.i./ha
(9.63 T/ha). Manju et al., (2018) reported maximum marketable fruit yield of $122 \mathrm{q} /$ ha in thiamethoxam 70 WS followed by Imidacloprid 70 WS (115 q/ha).

The phytotoxic effect of thiamethoxam $25 \%$ WGon okra variety Ajeet -333 revealed that okra plants sprayed with thiamethoxam $25 \%$ WG each at 25, 50 and 100 g a.i.ha ${ }^{-1}$ doses did not show any phytotoxic effects like epinasty, hyponasty, leaf injury, wilting, vein clearing and necrosis.

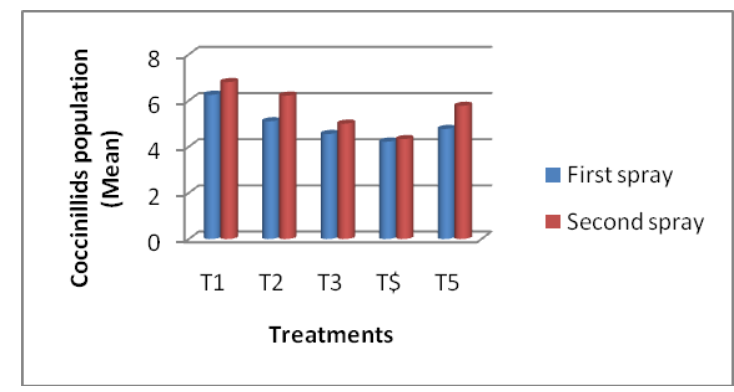

\footnotetext{
T1 Thiamethoxam 25\%WG@ 15 T4 Thiamethoxam 25\%WG@ 75 g a.i ha ${ }^{-1} \quad$ g a.i ha

Thiamethoxam 25\%WG@25 T5 Thiamethoxam 25 \%WG g a.i ha (Willoxam)@25g a.i ha

Thiamethoxam 25\%WG@50 T6 Untreated control

g a.i ha
}

Figure 3. Effect of thiamethoxam 25 WG on coccinellids in okra eco system (Pooled mean of two seasons)

The coccinellid population prior to application of insecticides ranged from 5.50 to 6.67 per ten plants (Table 4). Significant differences were observed in coccinellid population among the treatments after 
insecticidal application. The mean population of coccinellids after first spray, the plots treated with thiamethoxam 25 WG @ 15 g a.i. ha-1 recorded 6.25 coccinellids per ten plants followed by thiamethoxam 25 WG @ 25 g a.i. ha ${ }^{-1}$ (5.10 nos. 10 plants $^{-1}$ ). The standard check, Willoxam (Thiamethoxam 25 WG) @ 25 g.a.i. ha ${ }^{-1}$ recorded 4.77 coccinellids per 10 plants. Whereas, the plots maintained as untreated control harbored 8.33 coccinellids per 10 plants. After two rounds of spray, the plots treated with thiamethoxam 25 WG @ 15 g a.i. ha ${ }^{-1}$ recorded a mean coccinellid population per ten plants of 6.80 followed by thiamethoxam 25 \% WG @ 25 g a.i. ha ${ }^{-1}$ (6.21 nos. 10 plants $\left.^{-1}\right)$. The higher dose of thiamethoxam 25 WG @ 75 ga.i. ha ${ }^{-1}$ recorded mean population of 4.33 nos. 10 plants $^{-1}$ and the standard check, Willoxam (thiamethoxam 25 WG) @ 25 g.a.i. ha $^{-1}$ recorded 5.77 nos. 10 plants $^{-1}$. The untreated control recorded the highest coccinellid population of 12.33 per ten plants (Fig.3). Moscardini et al. (2015) reported that thiamethoxam used as seed treatments in sunflower fields negatively affected ladybeetles (Coleomegilla maculata and Hippodamia convergens). Zala et al. (2015) reported that Thiamethoxam 25WG@ 0.0125\% was relatively safer to the activity of spiders in okra ecosystem by recording the highest population

From the present study, it was found that thiamethoxam 25WG @ 25 g a.i.ha ${ }^{-1}$ is highly effective against sucking pest (aphids, leaf hopper and whitefly) of okra besides registering less harm to the predatory coccinellids. Thus, thiamethoxam 25WG @ 25 g a.i.ha-1 can be the potent component in IPM module for okra.

\section{CONCLUSION}

The results of the two field experiments conducted to assess the efficacy of thiamethoxam against the sucking pests on okra revealed that, Thiamethoxam 25\% WG 25 g a.i./ha (84.71-91.73, 94.12 - 98.11\% reduction over control) was highly effective against aphid, whitefly and leaf hoppers with was on par with with 50 ga.i./ha (64.28-76.90, 83.70 - 87.92\% reduction over control) and $75 \mathrm{~g}$ a.i./ha (73.4881.26 and $85.26-92.42 \%$ reduction over control) after first and second spray, respectively. Hence Thiamethoxam 25\% WG $25 \mathrm{~g}$ a.i./ha is sufficient and may be as field recommended does to suppress the sucking pests in okra.

\section{REFERENCES}

Anitha, K.R. and B.S. Nandihalli. 2009. Bioefficacy of newer insecticides against leafhopper and aphid in okra. Karnataka Journal Agricultural Sciences. 22(3): 714-715.

Awaneeshkumar., S.K., Sachan, Sudhir Kumar and Promish Kumar. 2017. Efficacy of some novel insecticides against whitefly Bemisia tabaci
(Gennadius) in brinjal. Journal of Entomology and Zoology Studies. 5(3): 424-427.

Bhatt, B., Karnatak, A.K. and Shivashankara. 2018. Bioefficacy of insecticides against aphids, whitefly and their predators on okra agroecosystem. Journal of Pharmacognosy and Phytochemistry. SP5: $40-45$

Bade, B.A., Nimbalkar, N.A., Kharbade, S.B. and A.S. Patil, 2017. Seasonal Incidence and Bioefficacy of Newer Insecticides and Biopesticide against Aphids on Okra and Their Effect on Natural Enemies, Int. J. Pure App. Biosci. 5(3): 1035-1043.

Gaikwad, B.B., Shetgar S.S., Sonkamble, M.M, Bhosle, A.B., and S.T. Shinde. 2014.Efficacy of different insecticides against population of lady bird beetle on safflower. Journal of Entomological Research. 38(2):129-130.

Ghosh, J., Chaudhuri, N and G. Roy. 2016. Department Bio-efficacy of thiamethoxam 25\% WG against sucking pests of okra under terai region of West Bengal. International Journal of Science, Environment and Technology. 5(3): 1217 - 1225.

Hemadri, T., L Vijaykumar., G Somu and Sharanabasava. 2018. Management of leafhopper, Amrasca biguttula biguttula (Hemiptera: Cicadellidae) in okra (Abelmoschus esculentus) through new insecticide molecules. International Journal of Chemical Studies, 6(2): 687-690.

Jiangong Jiang., Zhengqun Zhang., Jin Lin., Feng Liu and Wei Mu. 2019. The minimally effective dosages of nitenpyram and thiamethoxam seed treatments against aphids (Aphis gossypii Glover) and their potential exposure risks to honeybees (Apis mellifera). Sci Total Environ., 666(20): 68-78.

Manju, K.P., Vijaya Lakshmi, K.B., Sarath Babu and K. Anitha. 2018. Management of whitefly, Bemisia tabaci and Whitefly Transmitted Okra Yellow Vein Mosaic Virus (OYVMV) in Okra Int.J.Curr.Microbiol. App.Sci., Sp 6: 1676-1681.

Moscardini, V.F., Gontijo, P.C., Michaud, J.P. and G.A. Carvalho. 2015. Sublethal effects of insecticide seed treatments on two nearctic lady beetles (Coleoptera: Coccinellidae). Ecotoxicology. 24: 1152-1161.

Karthik, P., Sheela Venugopal, Datchinamurthy, K., Lokesh, S., Karthika, G., Sharmila, U., Paramasivam, M., Senguttuvan, K., Gunasekaran, $\mathrm{K}$ and S. Kuttalam. 2015. Bioefficacy, phytotoxicity, safety to natural enemies and residue dynamics of imidacloprid 70 WG in okra (Abelmoschus esculenta (L) Moench) under open field conditions. Crop Protection. 71: 88-94.

Patil, S. R., Lande, G.K., Awasthi, S. and U.P. Barkhade. 2014. Effect of different doses of newer insecticides against sucking pests of okra. Bioscan, 9(4):1597-1600.

Pawar, S.A., Zanwar, P.R., Lokare, S.G., Dongarjal, R.P. and M.M. Sonkamble. 2016. Efficacy of newer insecticides against sucking pests of okra. Indian Journal of Entomology, 78(3): 257-259.

Prem Kumar, K.N. and Ashwanikumar. 2017. Efficacy of Selected Insecticides against Sucking Insect 
Pests [Amrasca biguttula biguttula (Ishida) and Bemisia tabaci (Gennadius)] of Okra [Abelmoschus esculentus (L.) Moench]. Int.J.Curr.Microbiol.App. Sci., 6(8): 3256-3259.

Sagar Anand Pandey. 2018. Relative bio-efficacy of newer insecticide molecules against okra shoot and fruit borer and their dissipation: A review. Journal of Pharmacognosy and Phytochemistry, 7(3): 93-104.

Thangachamy, P., Karthik, P., Sanjeevi kumar, A., and K. Bharathidasan. 2015. Evaluation of thiamethoxam $25 \%$ wg against sucking pests in okra. Annals of plant and soil research. $17 \mathrm{sp} .343$ - 346.

Venkataravanappa, V., Krishnareddy, M., Lakshinimarayan reddy, C.N and Salil Jalali. 2012. Managament of okra YVM disease through neem product and insecticides. Annals of Plant Protection Science. 19(2): 487-488.
Wettstein, F.E., Kasteel, R., Garcia, D.M.F., Hanke, I., Huntscha, S., Balmer, M.E., Poiger, T., and T.D. Bucheli. 2016. Leaching of the neonicotinoids thiamethoxam and imidacloprid from sugar beet seed dressings to subsurface tile drains. J. Agric. Food Chem. 64: 6407-6415.

Zala, N.B., Nikoshe, A.P. and T.M. Bharpoda. 2015. Relative impact of insecticidal application on population of natural enemies in okra. Bioscan. 10(3): 955-958p.

Zhang, Z., Zhang, X., Wang, Y., Zhao, Y., Lin, J., Liu, F., and W. Mu. 2016. Nitenpyram, dinotefuran, and thiamethoxam used as seed treatments act as efficient controls against Aphis gossypii via high residues in cotton leaves. J. Agric. Food Chem. 64: 9276. 